\title{
DACBench: A Benchmark Library for Dynamic Algorithm Configuration
}

\author{
Theresa Eimer $^{1}$, André Biedenkapp ${ }^{2}$, Maximilian Reimer ${ }^{1}$, Steven Adriaensen ${ }^{2}$, \\ Frank Hutter $^{2,3}$ and Marius Lindauer ${ }^{1}$ \\ ${ }^{1}$ Information Processing Institute (tnt), Leibniz University Hannover, Germany \\ ${ }^{2}$ Department of Computer Science, University of Freiburg, Germany \\ ${ }^{3}$ Bosch Center for Artificial Intelligence, Renningen, Germany \\ \{eimer, reimerm, lindauer\}@tnt.uni-hannover.de, \{biedenka, adriaens, fh $\} @$ cs.uni-freiburg.de
}

\begin{abstract}
Dynamic Algorithm Configuration (DAC) aims to dynamically control a target algorithm's hyperparameters in order to improve its performance. Several theoretical and empirical results have demonstrated the benefits of dynamically controlling hyperparameters in domains like evolutionary computation, AI Planning or deep learning. Replicating these results, as well as studying new methods for DAC, however, is difficult since existing benchmarks are often specialized and incompatible with the same interfaces. To facilitate benchmarking and thus research on DAC, we propose DACBench, a benchmark library that seeks to collect and standardize existing DAC benchmarks from different AI domains, as well as provide a template for new ones. For the design of DACBench, we focused on important desiderata, such as (i) flexibility, (ii) reproducibility, (iii) extensibility and (iv) automatic documentation and visualization. To show the potential, broad applicability and challenges of DAC, we explore how a set of six initial benchmarks compare in several dimensions of difficulty.
\end{abstract}

\section{Introduction}

In the last years, algorithm configuration [Ansótegui et al., 2009; Hutter et al., 2011; López-Ibáñez et al., 2016] and in particular automated machine learning [Shahriari et al., 2016; Hutter et al., 2019] offered automatic methods optimizing the settings of hyperparameters to improve the performance of algorithms. However, practitioners of different communities have already known for a while that static hyperparameter settings do not necessarily yield optimal performance compared to dynamic hyperparameter policies [Senior et al., 2013]. One way of formalizing dynamic adaptations of hyperparameters is dynamic algorithm configuration (DAC) [Biedenkapp et al., 2020]. DAC showed its promise by outperforming other algorithm configuration approaches, e.g., choosing variants of CMA-ES [Vermetten et al., 2019] or dynamically adapting its step-size [Shala et al., 2020], dynamically switching between heuristics in AI planning [Speck et al., 2021], or learning learning rate schedules for computer vision [Daniel et al., 2016].
These results, however, also revealed a challenge for the further development of DAC. Compared to static algorithm configuration [Ansótegui et al., 2009; Hutter et al., 2011; López-Ibáñez et al., 2016], applying DAC also requires (i) the definition of a configuration space to search in, (ii) instances to optimize on and (iii) a reward signal defining the quality of hyperparameter settings. However, the optimizer and the algorithm to be optimized have to be integrated much closer in DAC. The current state of the algorithm and the reward function, for example, need to be queried by the optimizer on a regular basis and the applied hyperparameter changes need to be communicated to the algorithm. Therefore, creating reliable, reusable and easy-to-use DAC benchmarks is often fairly hard with no existing standard thus far.

This disparity between benchmarks in addition to the difficulty in creating new ones presents a barrier of entry to the field. Researchers not well versed in both target domain and DAC may not be able to reproduce experiments or understand the way benchmarks are modelled. This makes it hard for pure domain experts to create a DAC benchmark for their domain, severely limiting the number of future benchmarks we can expect to see. A lack of standardized benchmarks, in turn, will slow the progress of DAC going forward as there is no reliable way to compare methods on a diverse set of problems.

To close this gap, we propose DACBench, a suite of standardized benchmarks ${ }^{1}$. On one hand, we integrate a diverse set of AI algorithms from different domains, such as AI planning, deep learning and evolutionary computation. On the other hand, we ensure that all benchmarks can be used with a unified easy-to-use interface, that allows the application of a multitude of different DAC approaches as well as the simple addition of new benchmarks. This paper details the concepts and ideas of DACBench, as well as insights from the benchmarks themselves. Specifically, our contributions are:

1. We propose DACBench, a DAC benchmark suite with a standardized interface and tools to ensure comparability and reproducibility of results;

2. We discuss desiderata of creating DAC benchmarks and how we took them into account in DACBench;

3. We propose a diverse set of DAC benchmarks from different domains showing the breadth of DAC's potential,

${ }^{1}$ The project repository can be found at
https://github.com/automl/DACBench


allowing future research to make strong claims with new DAC methods;

4. We show that our DAC benchmarks cover different challenges in DAC application and research.

With this, we strive to lower the barrier of entrance into DAC research and enable research that matters.

\section{Related Work}

DAC is a general way to formulate the problem of optimizing the performance of an algorithm by dynamically adapting its hyperparameters, subsuming both algorithm configuration (AC) [Hutter et al., 2017] and per-instance algorithm configuration (PIAC) [Ansótegui et al., 2016]. While AC methods can achieve significant improvements over default configurations PIAC algorithms have demonstrated that searching for a configuration per instance can further improve performance. In a similar way, DAC can navigate the over time changing search landscape in addition to instance-specific variations.

Theoretically, this has been shown to be optimal for the $(1+(\lambda, \lambda))$ genetic algorithm [Doerr and Doerr, 2018], and to enable exponential speedups compared to AC on a family of AI Planning problems [Speck et al., 2021].

Empirically, we have seen dynamic hyperparameter schedules outperform static settings in fields like Evolutionary Computation [Shala et al., 2020], AI Planning [Speck et al., 2021] and Deep Learning [Daniel et al., 2016]. In addition, hyperheuristics [Ochoa et al., 2012] can also be seen as a form of DAC. In this field, it has been shown that dynamic heuristic selection outperforms static approaches on combinatorial optimization problems like Knapsack or Max-Cut [Almutairi et al., 2016].

In the context of machine learning, dynamically adjusting an algorithm's hyperparameters can be seen as a form of learning to learn where the goal is to learn algorithms or algorithm components like loss functions [Houthooft et al., 2018], exploration strategies [Gupta et al., 2018] or completely new algorithms [Andrychowicz et al., 2016]. While DAC does not attempt to replace algorithm components with learned ones, the hyperparameter values of an algorithm are often instrumental in guiding its progress. In some cases they become part of the algorithm. Dynamic step size adaption in ES using heuristics, for example, is very common, but can be replaced and outperformed by more specific DAC hyperparameter policies [Shala et al., 2020].

In other meta-algorithmic areas, reliable and well engineered benchmark libraries also facilitated research progress, incl. ASLib [Bischl et al., 2016], ACLib [Hutter et al., 2014], tabular NAS benchmarks (e.g. [Ying et al., 2019]) and HPOlib [Eggensperger et al., 2013]. In particular, DACBench is strongly inspired by HPOlib and OpenAI gym [Brockman et al., 2016] which also provide a unified interface to benchmarks. Although the hyflex framework [Ochoa et al., 2012] addresses a similar meta-algorithmic problem, in DACBench, we can model more complex problems (i.e., continuous and mixed spaces instead of only categoricals), consider state features of algorithms and cover more AI domains (not only combinatorial problems).
Furthermore DACBench is designed to build upon existing benchmark libraries in target domains by integrating their algorithm implementations. This includes well-established benchmarks like COCO [Hansen et al., 2020] or IOHProfiler [Doerr et al., 2018].

\section{Formal Background on DAC}

DAC aims at improving a target algorithm's performance through dynamic control of its hyperparameter settings $\lambda \in \Lambda$. To this end, a DAC policy $\pi$ queries state information $s_{t} \in \mathcal{S}$ of the target algorithm at each time point $t$ to set a hyperparameter configuration: $\pi: \mathcal{S} \rightarrow \Lambda$. Given a starting state $s_{0}$ of the target algorithm, a maximal number of solving steps $T$, a probability distribution $p$ over a space of problem instances $i \in \mathcal{I}$, and a reward function $r_{i}: \mathcal{S} \times \Lambda \rightarrow \mathbb{R}$ depending on the instance $i$ at hand, the objective is to find a policy maximizing the total return:

$$
\int_{\mathcal{I}} p(i) \sum_{t=0}^{T} r_{i}\left(s_{t}, \pi\left(s_{t}\right)\right) \mathrm{d} i
$$

Following [Biedenkapp et al., 2020], one way of modelling this task is as a contextual MDP $M_{\mathcal{I}}=\left\{M_{i}\right\}_{i \sim \mathcal{I}}$ [Hallak et al., 2015], consisting of $|\mathcal{I}|$ MDPs. Each $M_{i}$ represents one target problem instance $i$ with $M_{i}=\left(\mathcal{S}, \mathcal{A}, \mathcal{T}_{i}, r_{i}\right)$. This formulation assumes that all $M_{i}$ share a common state space $\mathcal{S}$, describing all possible algorithm states, as well as a single action space $\mathcal{A}$ choosing from all possible hyperparameter configurations $\Lambda$. The transition function $\mathcal{T}_{i}: \mathcal{S} \times \mathcal{A} \rightarrow \mathcal{S}$, corresponding to algorithm behaviour, and reward function $r_{i}$, however, vary between instances.

This formulation allows to apply different configuration approaches on the same problem setting, e.g., algorithm configuration by ignoring all state information $(\pi: \emptyset \rightarrow \Lambda)$, per-instance algorithm configuration by only taking the instance into account $(\pi: \mathcal{I} \rightarrow \Lambda)$ or a full DAC agent $(\pi: \mathcal{S} \times \mathcal{I} \rightarrow \Lambda)$ on the contextual MDP (information about $i \in \mathcal{I}$ is typically directly reflected in $s \in \mathcal{S}$ ). In view of how close this DAC formulation is to reinforcement learning (RL), in the remainder of the paper we will continue to refer to hyperparameter settings as actions and hyperparameter schedules as policies. Nevertheless, we consider DAC as a general problem that can be solved in different ways, incl. supervised learning, reinforcement learning or even hand-designed policies, e.g., cosine annealing for learning rate adaption in deep learning [Loshchilov and Hutter, 2017] or CSA for CMAES [Chotard et al., 2012].

\section{DACBench}

With DACBench, we strive for an easy-to-use, standardized and reproducible benchmark library that allows evaluating DAC on several, diverse benchmarks. To this end, we will first describe which components are needed to define a DAC benchmark, see Figure 1, and then explain how we can make use of it to ensure our design objectives.

\subsection{Components of a DAC Benchmark}

Inspired by the flexibility that the modelling as a cMDP allows and the success of OpenAI's gym environments, each 


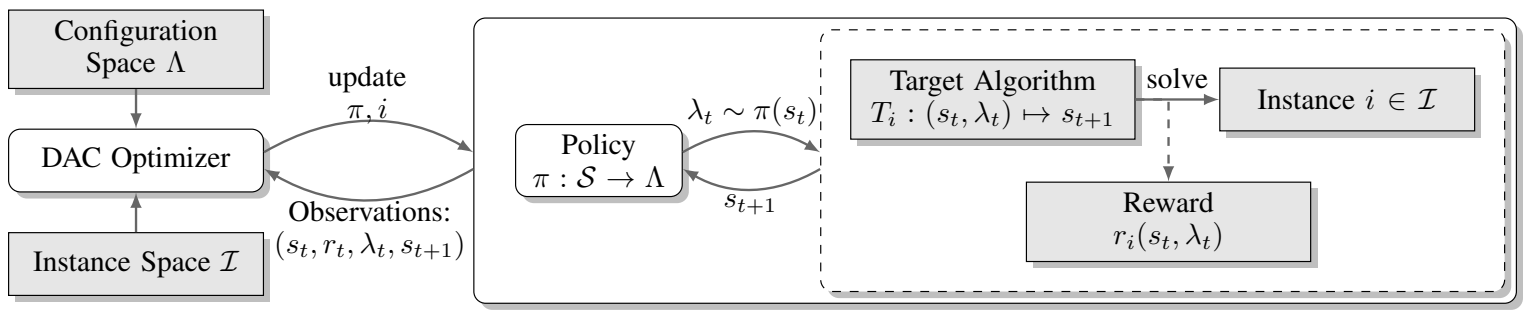

Figure 1: Interaction between optimizer, policy and all components of a DAC benchmark; latter in grey boxes.

DACBench benchmark is modelled along these lines, with the following benchmark-specific design decisions.

Action Space $\mathcal{A}$ describes ways of modifying the current configuration. In the simplest case, the action space directly corresponds to the hyperparameter space, incl. all hyperparameter names and the corresponding ranges.

State Space $\mathcal{S}$ describes available information about the target algorithm state. This can be enriched by context information about the instance at hand. We recommend that it is (i) cheap-to-compute information that is (ii) available at each step and (iii) measures the progress of the algorithm.

Target Algorithm with Transition Dynamics $\mathcal{T}_{i}$ implicitly defines which states $s_{t+1}$ are observed after hyperparameter configuration $\lambda_{t}$ is chosen in state $s_{t}$. It is important to fix the target algorithm implementation (and all its dependencies) to ensure that this is reproducible. An implicit design decision of a benchmark here is how long an algorithm should run before the next step description is returned.

Reward Function $r_{i}$ provides a scalar signal of how well the algorithm can solve a given instance. It is an analogue to the cost function in AC and PIAC and should be the optimization target, e.g., prediction error, runtime or solution quality.

Instance Set $\mathcal{I}$ defines variants of a given problem that has to be solved s.t. the learned policy is able to generalize to new, but similar instances. ${ }^{2}$ To assess generalization performance, a training and test set of instances is required. In addition, instances can be described by instance features [Bischl et al., 2016] which facilitates learning of per-instance policies.

This fine granular view on benchmarks allows us on one hand to create a multitude of different benchmarks, potentially with different characteristics. On the other hand, a benchmark in DACBench is a specific instantiated combination of these components s.t. DACBench contributes to reproducible results.

\subsection{Practical Considerations \& Desiderata}

DACBench provides a framework to implement the design decisions above with a focus on accessibility, reproducibility and supporting further research on DAC.

Accessibility So far, applying a new DAC optimizer to a target problem domain requires domain knowledge to be able to interface with a potential algorithm. Comparing optimizers

\footnotetext{
${ }^{2}$ For simplicity, we only discuss the case of a set of training instances. In general, DACBench also supports instance generators s.t. the set of instances does not have to be fixed in advance.
}

across multiple benchmarks of varying characteristics often requires re-implementing or adapting parts of the optimizers to fit the different interfaces, hurting the consistency of the comparison and taking a lot of time and effort.

Similarly, developing and providing new and interesting benchmarks is challenging as, without a standardized interface, there is little guidance on how to do so. Thus, domain experts wanting to provide a DAC benchmark of a target algorithm often construct their own interface, which can be timeconsuming even with a background in MDPs.

Providing a standardized interface would alleviate the issues and facilitate moving DAC as a field forward. Therefore, DACBench provides a common interface for benchmarks, based on OpenAI's gym API [Brockman et al., 2016], that makes interaction with DAC optimizers as simple as possible. This interface is lightweight and intuitive to implement for experts from different domains, encouraging collaboration in the creation of new benchmarks and optimizers. It also allows domain experts to modify existing benchmarks with little effort and minimal knowledge of the base code to create new and interesting variations of known benchmarks, see Appendix C.

Reproducibility As discussed before, adapting an algorithm for DAC can be challenging as there are many design decisions involved. On one hand, to allow studies of new DAC characteristics, we believe it is important to give researchers the flexibility to adjust these components. Therefore, we do not want to propose a framework that fixes too many decision points as it could restrict important future research. On the other hand, we believe there is a need for standardized benchmarks to facilitate comparing different methods as well as reproducing research results. For this purpose, all design decisions of the original experiments should be reproducible. To this end, DACBench includes a mechanism to customize as many of these design decisions as possible, but also to record them such that other researchers can reproduce the experiments (for more details, see Appendix A).

Facilitating Further Research Lastly, DACBench supports researchers by providing resources needed to work on DAC problems as well as thorough documentation of the design decisions of each benchmark. As existing benchmarks are often not documented very well, working with them requires thorough study of the code base. Instead, DACBench provides all important details about individual benchmarks in a concise manner through comprehensive documentation.

Furthermore, DACBench provides quality of life components like structured logging and visualization that make working with DACBench seamless. The logging system gives 
users the option to save a variety of details like the policies or state information for later analysis. Further, the built-in visualization tools make evaluating experiments easy (examples include Figures 3, 3 and 5) and can directly use the data provided by the logging system.

These considerations contribute to driving open research on DAC forward by ensuring easy reproducibility of experiments, usability for a diverse audience and sharing of experiment configurations. By adopting a simple yet modular interface, we improve general accessibility to the field as well as the ability to continuously evolve DAC benchmarks.

\subsection{Six Initial Diverse DAC Benchmarks}

We propose six initial benchmarks for DACBench from different domains and with different challenges (for in-depth descriptions, see Appendix B). We believe these present a versatile set of problems both for testing DAC methods across diverse benchmarks and developing new approaches.

Sigmoid \& Luby [Biedenkapp et al., 2020] are time series approximation tasks with no underlying target algorithm. These artificial benchmarks run very quickly, their optimal policies can be computed efficiently for all possible instances (i.e. transformations of the functions themselves) and it is easy to generate instance sets for a wide range of difficulties. Therefore, Sigmoid and Luby are ideal for DAC developers, e.g. to verify that agents can learn the optimal policy or slowly ramp up the instance heterogeneity in order to test its generalization capabilities.

FastDownward [Helmert, 2006] is a state-of-the-art AI Planner, which gives rise to a more complex benchmark. The task here is to select the search heuristic at each step on a specific problem family with two complementary heuristics. This can be considered one of the easier benchmarks even though significant performance gains on competition domains are possible with four commonly used heuristics [Speck et al., 2021]. The basic instance set we provide includes optimal policy information as an upper performance bound.

CMA-ES [Hansen et al., 2003] is an evolutionary strategy, where the DAC task is to adapt the algorithm's steps size [Shala et al., 2020] when solving BBOB functions. However, finding a good solution in this continuous space is potentially harder than the discrete heuristic selection in FastDownward. While optimal policies are unknown for this benchmark, there is a strong established dynamic baseline in CSA [Chotard et al., 2012].

ModEA includes an example of dynamic algorithm selection for variants of CMA-ES on BBOB functions [Vermetten et al., 2019]. In contrast to the CMA-ES benchmark, a combination of 11 EA elements with two to three options each are chosen in each step; this combination makes up the final algorithm. This multi-dimensional, large action space makes the problem very complex. So we expect this to be a hard benchmark, possibly too hard for current DAC approaches to efficiently determine an effective DAC policy.

SGD-DL adapts the learning rate of a small neural network learning a simple image classification task [Daniel et al., 2016]. The small network size allows for efficient development and benchmarking of new DAC approaches. By

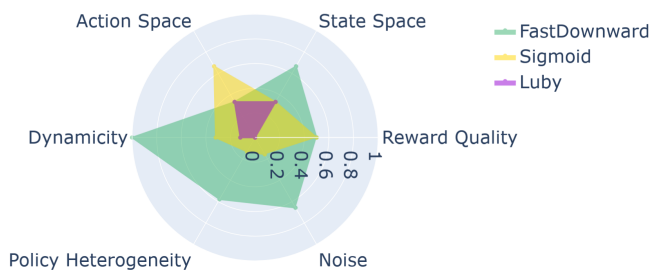

(a)

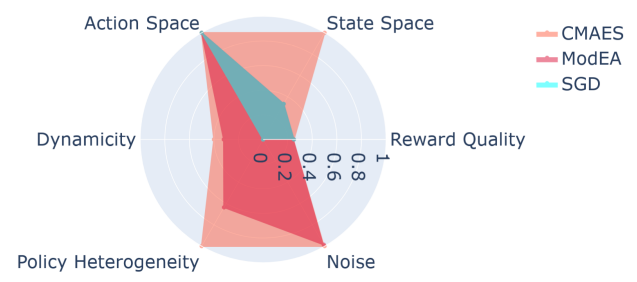

(b)

Figure 2: Ranked comparison of difficulty dimensions in DACBench benchmarks. Lower values correspond to easier characteristics.

varying the instance (dataset-seed pairs) and the network architecture, this benchmark nevertheless opens up ample possibility to grow ever harder as DAC advances.

\section{Empirical Insights Gained from DACBench}

In order to study our benchmarks, we discuss dimensions of difficulty which are relevant to the DAC setting. To provide insights into how our benchmarks behave in these dimensions, we use static policies, known dynamic baselines and random dynamic policies to explore their unique challenges.

\subsection{Setup}

To show how our benchmarks behave in practice, we mainly use the static and random policies built into DACBench and, where possible, make use of optimal policies. All of them were run for 10 seeds with at most 1000 steps on each instance. For benchmarks with a discrete action space, static policies cover all the actions. The two benchmarks with continuous action spaces, CMA-ES and SGD-DL were run with 50 static actions each, distributed uniformly over the action space. For details on the hardware used, refer to Appendix D.

\subsection{Coverage of Difficulty Dimensions}

Similar to Biedenkapp [2020], we identified six core challenges of learning dynamic configuration policies to characterize our benchmarks. For comparison's sake, we define a scale for each attribute and measure these on our benchmarks. These dimensions of difficulty are: (i) State and (ii) action space size increase the difficulty of the problem by varying information content, requiring the agent to learn what state information is relevant and which regions in the action space are useful. (iii) Policy heterogenity quantifies how successful different policies are across all instances. (iv) Reward quality refers to the information content of the given reward signal. (v) Noise can disturb the training process through noisy 

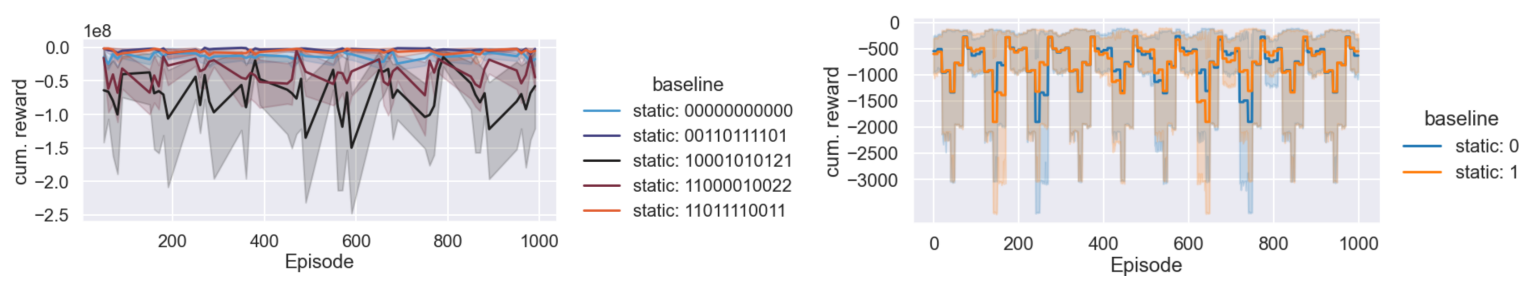

Figure 3: Left: Performance of 5 static ModEA policies with $95 \%$ confidence interval. The legend shows which components of ModEA were used. Right: Comparison of average performance of static FastDownward policies with $95 \%$ confidence interval.

transitions or rewards. Lastly, (vi) dynamicity shows how frequently the action should be changed, i.e. how complex wellperforming policies need to be. See Appendix E for details.

Figure 2 shows how the benchmarks compare with respect to these dimensions of difficulty. While the reward quality is not fully covered, we cover all other dimensions well, with at least a very, moderately and not especially difficult benchmark in each. Additionally, all DACBench benchmarks show a different profile. The data shows that Luby could be considered the easiest of the six, with little noise or policy heterogeneity and a relatively low dynamicity score, requiring only infrequent action changes. SGD-DL's footprint looks similar, though its continuous action space makes for a difficulty spike in that category. While Sigmoid's reward function obscures quite a bit of information, it is not very difficult in the other dimensions. FastDownward on the other hand leads the dynamicity dimension by far, showing a need for more active control. It is also fairly challenging with regard to noise and policy heterogeneity. CMA-ES is even more difficult in these, while also having the largest state space. A more informative reward and lower dynamicity contrast it and other benchmarks. ModEA's difficulty, on the other hand, seems similar except for the challenge of a continuous state space.

While this shows that our benchmark set covers all of our dimensions of difficulty with the exception of reward quality fairly well, we will continue to explore the dimensions of noise, policy heterogeneity and dynamicity in greater detail in order to give a more detailed impression of how these dimensions are expressed.

\subsection{Degree of Randomness}

To show how randomness is expressed in our benchmarks, we investigate its effects on FastDownward and ModEA.

We quantified randomness by using the standard deviation of the cumulative reward between different seeds for the same actions, each repeated 10 times. ModEA was one of the benchmarks that had a very high relative standard deviation and thus a very high noise score, see Figure 3. While static policies from different parts of the action space vary in performance, their confidence intervals grow much larger the worse they perform. This is to be expected, as policies with a high reward have found EA components that quickly find the optimal solution of the black-box function at hand. If the resulting EA cannot find a solution quickly, the individuals in each generation will have very different proposed solutions, thus resulting in unstable performance. So even though ModEA contains quite a bit of noise, the noise is heteroscedastic, i.e., it is not evenly distributed across the policy space, providing an additional challenge.

FastDownward, on the other hand, also has a high rating in the noise category, but the way its noise is distributed is quite different, see Figure 3. W.r.t. the average performance of both static policies, the $95 \%$ confidence interval is up to twice as large as the performance value itself. In contrast to ModEA, the noise is large but likely homoscedastic.

\subsection{Effect of Instances}

To investigate the effect instances have on our benchmarks, we examine CMA-ES, which showed the highest policy heterogeneity above, and Sigmoid, for which we can compute the optimal policy. CMA-ES and ModEA both operate on instance sets comprised of different function classes between which we can clearly see very different behaviour. The Schaffers function (see Figure 4 left) illustrates that the handdesigned CSA is indeed a good dynamic policy; it outperforms all other static and random policies.

In contrast, CSA performs much worse on the Ellipsoid function (Figure 4 middle). Using the probability estimation proposed by [Shala et al., 2020] based on the Wilcoxon rank sum test, CSA's probability of outperforming any given static policy is $74.6 \%$ overall; also shown on a per-instance level in the algorithm footprint [Smith-Miles et al., 2014] in Figure 4. While this shows that CSA's dynamic control policy is preferred on most of CMA-ES instance space, there are also regions that require a different approach, underlining the importance of instance dependent methods.

On the Sigmoid benchmark we see that performance differences between instances persist even for the optimal policy (see Figure 5 left). While it performs very well on some instances, this is far from the case for all of them. Indeed, while it is possible to gain the best possible reward of 10 on some instances, there is an almost even distribution of rewards between the maximum and minimum cumulative reward.

Overall, different instances can have a significant influence on the overall performance, both in terms of which policies are successful on them and how well an agent can do.

\subsection{Is Dynamic Better than Static?}

Even though we have empirical evidence of DAC agents surpassing static baselines for all of our benchmarks [Daniel et al., 2016; Vermetten et al., 2019; Biedenkapp et al., 2020; Shala et al., 2020; Speck et al., 2021], we analyse and compare the performance of dynamic and static policies on our benchmarks. This way we can estimate the difficulty both in finding a good dynamic policy that surpasses a simple random one but also the difficulty of outperforming the static 

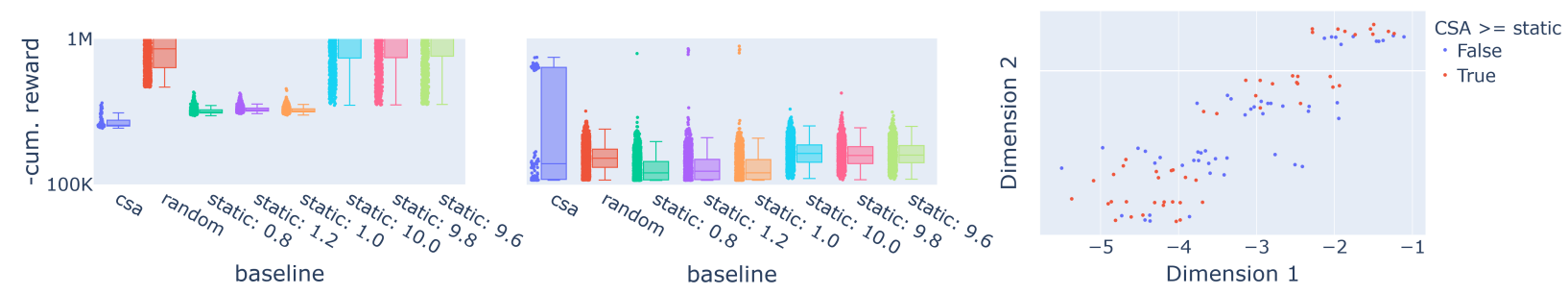

Figure 4: Policy evaluation of CMA benchmark on Schaffers (left) and Ellipsoid (middle) functions (with 3 best and worst static policies). Right: Algorithm footprint t-SNE plot of CMA-ES instances showing where CSA outperforms all static policies.
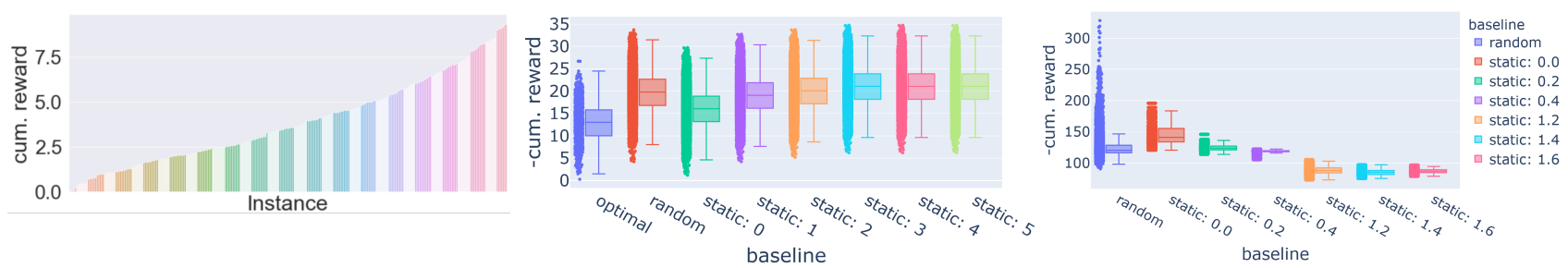

Figure 5: Left: Best possible reward for each sigmoid instance. Middle: Static and dynamic policies on Luby. The reward is 0 if the agent guesses the correct sequence element, -1 otherwise. Right: Static (best and worst 3 ) and dynamic policies on SGD-DL with $\lambda=10^{-x}$. The reward here is the validation loss (negative log-likelihood).

policies. Insights into the relationship between static and dynamic policies can highlight characteristics of a benchmark, give upper and lower performance bounds and show the effect size we can expect from DAC approaches in the future.

Our evaluation clearly shows that the benchmarks have a very different sensitivity to dynamic policies. In Luby (Figure 5 middle) we can see that the most common elements of the Luby sequence, elements one and two, outperform the dynamic random policy. As $50 \%$ of the Luby sequence consist of the first element and $25 \%$ of the second, this is the expected behaviour. Therefore it also makes sense that the optimal policy outperforms all other policies. The random policy does not perform very well, showing that there is a lot of room to improve over it and subsequently over the static policies.

Similarly, the random policy of SGD-DL outperforms some of the worst static policies on average, but does very poorly compared to them on many occasions (see Figure 5 right). Improving over the best static policies here will therefore be much harder for a DAC agent. This is also an example of the fact that dynamically adapting hyperparameters can outperform static settings, as [Daniel et al., 2016] showed for this setting, but the region of well-performing dynamic policies seem to be much smaller than for Luby above. This is the reason for the benchmark's low dynamicity rating. Unlike e.g. FastDownward, which favors frequent action changes regardless of their quality, SGD-DL requires a more subtle approach with more consistency and carefully selected actions.

Therefore, we believe dynamicity will play a large role in how DAC methods should approach benchmarks. While choosing a new action each step for SGD-DL can of course be learned successfully over time, it is a much harder task than Luby. Methods keeping actions for a number of steps at a time may have better success here [Vermetten et al., 2019].

\section{Conclusion}

We propose DACBench, a standardized benchmark suite for dynamic algorithm configuration (DAC). With it, we provide a framework to configure DAC benchmarks that both enables reproducibility and easy modifications, ensuring that DACBench can help evolve DAC benchmarks further. For example, we plan to extend the FastDownward benchmark beyond single domains and include existing instance features from e.g. Exploratory Landscape Analysis (ELA) for CMAES and ModEA. Furthermore, DACBench is easily extendable and we will add new benchmarks, developed by us and the community. As an incentive for researchers to tackle some of the most important difficulties in solving DAC, we provide challenges for several dimensions of hardness. In order to assist in developing these new approaches, we also include tools for tracking important metrics and visualization, making DACBench very easy to use without knowledge of the target domains. Overall, we believe DACBench will make DAC more accessible to interested researchers, make existing DAC approaches more easily comparable and provide a direction for research into new methods. For future work, we plan to build surrogate benchmarks, similar to [Eggensperger et al., 2018] for AC and [Siems et al., 2020] for NAS, to enable DAC benchmarking with minimal computational overhead and minimized $\mathrm{CO}_{2}$ footprint.

\section{Acknowledgements}

We thank Gresa Shala, David Speck and Rishan Senanayake for their contributions to the CMA-ES, FastDownward and SGD-DL benchmarks respectively. Theresa Eimer and Marius Lindauer acknowledge funding by the German Research Foundation (DFG) under LI 2801/4-1. All authors acknowledge funding by the Robert Bosch $\mathrm{GmbH}$. 


\section{References}

[Almutairi et al., 2016] A. Almutairi, E. Özcan, A. Kheiri, and W. Jackson. Performance of selection hyper-heuristics on the extended hyflex domains. In Proc. of ISCIS, 2016.

[Andrychowicz et al., 2016] M. Andrychowicz, M. Denil, S. G. Colmenarejo, M. W. Hoffman, D. Pfau, T. Schaul, and N. de Freitas. Learning to learn by gradient descent by gradient descent. In Proc. of NeurIPS, pages 3981-3989, 2016.

[Ansótegui et al., 2009] C. Ansótegui, M. Sellmann, and K. Tierney. A gender-based genetic algorithm for the automatic configuration of algorithms. In Proc. of CP'09, pages 142-157, 2009.

[Ansótegui et al., 2016] C. Ansótegui, J. Gabàs, Y. Malitsky, and M. Sellmann. Maxsat by improved instance-specific algorithm configuration. AIJ, 235:26-39, 2016.

[Biedenkapp et al., 2020] A. Biedenkapp, H. F. Bozkurt, T. Eimer, F. Hutter, and M. Lindauer. Dynamic Algorithm Configuration: Foundation of a New Meta-Algorithmic Framework. In Proc. of ECAI, pages 427-434, 2020.

[Bischl et al., 2016] B. Bischl, P. Kerschke, L. Kotthoff, M. Lindauer, Y. Malitsky, A. Frechétte, H. Hoos, F. Hutter, K. LeytonBrown, K. Tierney, and J. Vanschoren. ASlib: A benchmark library for algorithm selection. AIJ, pages 41-58, 2016.

[Brockman et al., 2016] G. Brockman, V. Cheung, L. Pettersson, J. Schneider, J. Schulman, J. Tang, and W. Zaremba. OpenAI Gym. CoRR, abs/1606.01540, 2016.

[Chotard et al., 2012] A. Chotard, A. Auger, and N. Hansen. Cumulative step-size adaptation on linear functions. In Proc. of PPSN, 2012.

[Daniel et al., 2016] C. Daniel, J. Taylor, and S. Nowozin. Learning step size controllers for robust neural network training. In Proc. of AAAI, 2016.

[Doerr and Doerr, 2018] B. Doerr and C. Doerr. Optimal static and self-adjusting parameter choices for the $(1+(\lambda, \lambda))$ genetic algorithm. Algorithmica, 80(5):1658-1709, 2018.

[Doerr et al., 2018] C. Doerr, H. Wang, F. Ye, S. van Rijn, and T. Bäck. Iohprofiler: A benchmarking and profiling tool for iterative optimization heuristics. arXiv e-prints:1810.05281, 2018.

[Eggensperger et al., 2013] K. Eggensperger, M. Feurer, F. Hutter, J. Bergstra, J. Snoek, H. Hoos, and K. Leyton-Brown. Towards an empirical foundation for assessing Bayesian optimization of hyperparameters. In NeurIPS Workshop on Bayesian Optimization in Theory and Practice (BayesOpt'13), 2013.

[Eggensperger et al., 2018] K. Eggensperger, M. Lindauer, H. H. Hoos, F. Hutter, and K. Leyton-Brown. Efficient benchmarking of algorithm configurators via model-based surrogates. Machine Learning, 107(1):15-41, 2018.

[Gupta et al., 2018] A. Gupta, R. Mendonca, Y. Liu, P. Abbeel, and S. Levine. Meta-reinforcement learning of structured exploration strategies. In Proc. of NeurIPS, pages 5307-5316, 2018.

[Hallak et al., 2015] A. Hallak, D. Di Castro, and S. Mannor. Contextual markov decision processes. CoRR, abs/1502.02259, 2015.

[Hansen et al., 2003] Nikolaus Hansen, Sibylle D. Müller, and Petros Koumoutsakos. Reducing the time complexity of the derandomized evolution strategy with covariance matrix adaptation (CMA-ES). Evolutionary Computing, 11(1):1-18, 2003.

[Hansen et al., 2020] N. Hansen, A. Auger, R. Ros, O. Mersmann, T. Tušar, and D. Brockhoff. COCO: A platform for comparing continuous optimizers in a black-box setting. Optimization Methods and Software, 2020.
[Helmert, 2006] M. Helmert. The fast downward planning system. JAIR, 26:191-246, 2006.

[Houthooft et al., 2018] R. Houthooft, Y. Chen, P. Isola, B. Stadie, F. Wolski, J. Ho, and Pieter Abbeel. Evolved policy gradients. In Proc. of NeurIPS, 2018.

[Hutter et al., 2011] F. Hutter, H. Hoos, and K. Leyton-Brown. Sequential model-based optimization for general algorithm configuration. In Proc. of LION, pages 507-523, 2011.

[Hutter et al., 2014] F. Hutter, M. López-Ibánez, C. Fawcett, M. Lindauer, H. Hoos, K. Leyton-Brown, and T. Stützle. AClib: a benchmark library for algorithm configuration. In Proc. of LION, pages 36-40, 2014.

[Hutter et al., 2017] F. Hutter, M. Lindauer, A. Balint, S. Bayless, H. Hoos, and K. Leyton-Brown. The configurable SAT solver challenge (CSSC). AIJ, 243:1-25, 2017.

[Hutter et al., 2019] F. Hutter, L. Kotthoff, and J. Vanschoren, editors. Automated Machine Learning: Methods, Systems, Challenges. Springer, 2019. Available for free at http://automl.org/book.

[López-Ibáñez et al., 2016] M. López-Ibáñez, J. Dubois-Lacoste, L. Perez Caceres, M. Birattari, and T. Stützle. The irace package: Iterated racing for automatic algorithm configuration. Operations Research Perspectives, 3:43-58, 2016.

[Loshchilov and Hutter, 2017] I. Loshchilov and F. Hutter. Sgdr: Stochastic gradient descent with warm restarts. In Proc. of ICLR, 2017.

[Ochoa et al., 2012] G. Ochoa, M. Hyde, T. Curtois, J. Rodríguez, J. Walker, M. Gendreau, G. Kendall, B. McCollum, A. Parkes, S. Petrovic, and E. Burke. Hyflex: A benchmark framework for cross-domain heuristic search. In Proc. of EvoCOP, 2012.

[Senior et al., 2013] A. Senior, G. Heigold, M. Ranzato, and K. Yang. An empirical study of learning rates in deep neural networks for speech recognition. In Proc. of ICASSP, 2013.

[Shahriari et al., 2016] B. Shahriari, K. Swersky, Z. Wang, R. Adams, and N. de Freitas. Taking the human out of the loop: A review of Bayesian optimization. Proceedings of the IEEE, 104(1):148-175, 2016.

[Shala et al., 2020] G. Shala, A. Biedenkapp, N. Awad, S. Adriaensen, M. Lindauer, and F. Hutter. Learning step-size adaptation in CMA-ES. In Proc. of PPSN, pages 691-706, 2020.

[Siems et al., 2020] J. Siems, L. Zimmer, A. Zela, J. Lukasik, M. Keuper, and F. Hutter. NAS-Bench-301 and the case for surrogate benchmarks for neural architecture search. CoRR, abs/2008.09777, 2020.

[Smith-Miles et al., 2014] K. Smith-Miles, D. Baatar, B. Wreford, and R. Lewis. Towards objective measures of algorithm performance across instance space. Comput. Oper. Res., 45:12-24, 2014.

[Speck et al., 2021] D. Speck, A. Biedenkapp, F. Hutter, R. Mattmüller, and M. Lindauer. Learning heuristic selection with dynamic algorithm configuration. In Proc. of ICAPS'21, August 2021.

[Vermetten et al., 2019] D. Vermetten, S. van Rijn, T. Bäck, and C. Doerr. Online selection of CMA-ES variants. In Proc. of GECCO. ACM, 2019.

[Ying et al., 2019] C. Ying, A. Klein, E. Christiansen, E. Real, K. Murphy, and F. Hutter. Nas-bench-101: Towards reproducible neural architecture search. In Proc. of ICML, 2019. 